\title{
DATABASE COMPILATION AND VIRTUAL SCREENING OF SECONDARY METABOLITES DERIVED FROM MARINE FUNGI AS EPIDERMAL GROWTH FACTOR RECEPTOR TYROSINE KINASE INHIBITORS
}

\author{
MEGA WATTY, REZI RIADHI SYAHDI, ARRY YANUAR* \\ Department of , Faculty of Pharmacy, Universitas Indonesia, Depok, Indonesia. Email: arry.yanuar@ui.ac.id \\ Received: 21 April 2017, Revised and Accepted: 13 July 2017
}

\begin{abstract}
Objective: Epidermal growth factor receptor (EGFR), a transmembrane protein with cytoplasmic kinase activity, transduces growth factor signaling from the extracellular space to the cell. EGFR downstream signaling increases proliferation and reduces apoptosis. Agents that are targeted at intracellular tyrosine kinase are tyrosine kinase inhibitor small molecules, which have a mechanism of action that affects adenosine triphosphate binding to the receptor. The exploration of bioactive compounds from marine materials, including marine fungi, has become a major interest lately for anticancer treatment.
\end{abstract}

Methods: In this research, a database was created and in silico screening was conducted using AutoDock and Vina to obtain potential marine fungi bioactive compounds as EGFR-tyrosine kinase (EGFR-TK) inhibitors, which act as antiproliferative agents on tumor cell growth.

Results: This research has concluded that the three marine fungi compounds with the lowest binding free energy, FU0015, FU0051, and FU0202, have great potential as inhibitors of EGFR-TK.

Conclusions: Three active compounds were identified as inhibitors of EGFR-TK, which were Fiscalin A, derived from Neosartorya paulistensis KUFC 7897 (FU0015); Aspergiolide B, derived from Aspergillus flavus (FU0051); and Sporothrix A, derived from Sporothrix sp. (FU0202).

Keywords: Marine fungi compounds, Epidermal growth factor receptor-tyrosine kinase, Virtual screening, AutoDock, Vina, Antiproliferative.

(C) 2017 The Authors. Published by Innovare Academic Sciences PvtLtd. This is an open access article under the CC BYlicense (http://creativecommons. org/licenses/by/4. 0/) DOI: http://dx.doi.org/10.22159/ajpcr.2017.v10s5.23118

\section{INTRODUCTION}

Epidermal growth factor receptor (EGFR) is an important target in the process of reducing tumor growth. More than $60 \%$ of non-small cell lung cancers express EGFR [1]. EGFR downstream signaling increases proliferation and angiogenesis and reduces apoptosis [2]. The overexpression of EGFR has been detected in the majority of human carcinomas [3].

Tyrosine kinase inhibitors (TKIs) are one of four classes of anti-EGFR agents used for cancer therapy [4]. In 2015, gefitinib (one of the TKIs) was approved by the Food and Drug Administration (FDA) as an inhibitor of EGFR [5]. In addition to synthetic drugs, natural products have an important role in cancer therapy. Chemical compounds from natural products can be potential new medicines. The exploration of bioactive compounds from marine materials, including marine fungi, has become a major activity lately for anticancer treatment. Currently, there are anticancer compounds from marine fungal metabolites that have entered clinical trials. One of the alkaloid compounds, plinabulin, is a modified structure based on the (-)-phenylahistin metabolites of the terrestrial and marine fungi Aspergillus ustus [6]. As the development of microbiology technology increases in the future, the availability of microorganisms such as marine fungi will increase and the costs of production will be reduced. Therefore, bioactive compounds of marine fungi have the potential to be new drug compounds [7]. A database of active compounds was created to ease in silico and in vitro research further.

In silico research can support conventional research (wet lab) that is relatively expensive and takes time [8]. AutoDock (Automatic Docking) is widely used to predict the complex of a biomolecular structure or to perform functional analysis and molecular design. AutoDock uses a grid-based method to rapidly evaluate the target protein's binding energy binding energy in search of a large conformation space for a large number of ligands around the protein. Visualization of active sites and the volume of searching space can be conducted through the AutoDockTools application [9]. AutoDock Vina (Vina) is a new program for virtual screening. Vina achieves greater speed than AutoDock 4, calculates the grid maps automatically and quickly, and does not need to be stored on a disk [10]. AutoDock and Vina are tools that can assist in the exploration of new drug compounds through virtual screening. In this research, the virtual screening of marine fungi bioactive compounds was conducted to find potential compounds for EGFR-tyrosine kinase (EGFR-TK) inhibitors. A database of marine fungi was created using a data mining method on literature about bioactive compound derived from marine fungi, whether known specifically as anticancer or not. Then, we explored whether those compounds can specifically inhibit the growth of lung cancer

To be reliable, virtual screening needs to be validated. Validation was performed by calculating the value of the root mean square deviation (RMSD), the enrichment factor (EF), and receiver operating characteristics (ROC). RMSD was used to interpret the protein model and the quality of the ligand positions as the docking result that affects molecule conformation [11]. EF is a virtual screening algorithm with parameters that was used to determine the amount of active compound contained in the database of compounds [12]. ROC was used to analyze the amount of active and inactive compounds from the database of screening results. Some compounds were configured into a calculation formula and represented in the form of curves [13]. The results obtained in this research were hits compounds that are expected to be developed further as novel inhibitors of EGFR-TK that plays an important role in the growth of lung cancer. Further in vitro research can be conducted to ensure the potency of those hits compounds as EGFR-TK inhibitors. 


\section{METHODS}

\section{Tools}

This research used the following computer hardware: A central processing unit (CPU; Intel ${ }^{\circledR}$ Xeon E5620), graphics processing unit (GPU; Nvidia ${ }^{\circledR}$ GeForce GTX 780), and 32 gigabytes (GB) of DDR3 random access memory (RAM) connected to the Internet. Supporting tools consisted of a monitor (AOC, China), mouse (Microsoft, China), and keyboard (HP, China). The software used included AutoDockTools, AutoDock 4.0, PyMOL, PyRx, MarvinSketch, Open Babel, LigandScout, and UCSF Chimera.

\section{Materials}

Dataset with a total of 268 three-dimensional compound structures from marine fungi were used as ligands and EGFR-TK was used as a receptor.

\section{Working methods \\ Preparation of the marine database}

To collect the three-dimensional structures of marine fungi, data mining was conducted on the literature related to marine fungi bioactive compounds and the chemical structures of those bioactive compounds were downloaded from PubChem and ChemSpider. If the three-dimensional structures could not be downloaded, they were created using MarvinSketch with the minimization parameters of the MMFF94 force field and a strict optimization limit. Various marine fungi bioactive compounds, whether known specifically as anticancer or not, were included in the marine database, resulting in 268 structures that were downloaded from PubChem and ChemSpider or created using MarvinSketch [14-33].

\section{Preparation of target macromolecules}

The target macromolecules (EGFR-TK) were downloaded from Protein Data Bank (PDB) with PDB ID "4WKQ" using inclusion criteria of wild type/non-mutant and exclusion criteria of macromolecules with a resolution higher than $2.5 \AA$ (Angstroms) and macromolecule with an incomplete chain. 4WKQ is the structure of EGFR, which binds to gefitinib. This protein is a monomer consisting of 330 amino acid (AA) residues. There are two similar subunits in the structure of the crystal, both Chain A and B with length of 330 AA residues. The $4 \mathrm{WKQ}$ crystal has a resolution of $1.85 \AA$ and occupies a volume of $14.4 \times 14.4 \times 14.4 \mathrm{~mm}[34]$. The macromolecules were separated from their solvent and ligand/non-standard residues. Then, optimization was conducted, which included the deletion of water molecules, the addition of hydrogen, the preparation of charges by adding Gasteiger partial charges, the administration of the AutoDock force field, and the implementation of minimization. The macromolecules were saved in *.pdb and then converted to *.pdbqt.

\section{Virtual screening}

The following parameters were evaluated using AutoDock and Vina before screening was performed. In AutoDock, the Lamarckian GA option was chosen, and the maximum number of energy evaluations was 10 for validation and 100 for virtual screening. In Vina, the number of modes option was 9 for validation and 100 for virtual screening, and exhaustiveness was 8. The virtual screening of the marine database against EGFR-TK was done using AutoDock and Vina. The virtual screening methods were validated using positive controls from the macromolecules' ligands and FDA-approved EGFR inhibitors. The FDAapproved drugs that were used consisted of afatinib, erlotinib, gefitinib, imatinib, lapatinib, and neratinib. The results of binding energy, inhibition constant, and molecule interaction were analyzed.

\section{Protein-ligand interaction}

The superposition and interaction of the database compounds against cocrystal ligands were inspected visually using LigPlot ${ }^{+}$, PyMOL, and LigandScout software. The aim of superposition visualization is to visualize the proximity of ligands from the screening results against the cocrystal ligands and positive control while the objective of interaction visualization is to visualize the interaction between ligands and AA residues.

\section{RESULTS AND DISCUSSION}

Coordinates for the target receptor (4WKQ) were determined using a grid box ( $x=2.436 ; y=193.257 ; z=21.771)$ searching method by focusing on the crystal ligand.

Virtual screening method optimization using AutoDock tools

The virtual screening method was optimized for the EGFR target by redocking the cocrystal to determine the optimum box size position. One target receptor was used for the virtual screening, EGFR. The virtual screening parameters were set to generate more negative binding energy values, more homogeneous cluster distributions, and RMSD with a value below $2 \AA$. Based on the results of the data, the RMSD of the EGFR target receptor for a $60 \times 60 \times 60$ box reached the criteria (2.113 from the * .dlg file), so it can be used for further virtual screening. The value of the cocrystal ligand binding energy was $-7.43 \mathrm{kcal} / \mathrm{mol}$ and the value of the inhibition constant was $3.56 \mu \mathrm{M}$. The ideal position change was within $<2 \AA$. If a molecule has a value of RMSD $>2 \AA$, it indicates that there was a far enough shift of molecules [11]. Fig. 1 shows the further analysis of cocrystal superposition before and after the virtual screening.

\section{Virtual screening method validation}

RMSD, EF, and ROC are validation criteria. The grid box from the optimization result was used $(60 \times 60 \times 60$ units $)$. Validation was conducted to ascertain whether the optimum box for virtual screening generates a valid result for each validation parameter as well as to verify whether macromolecules and cocrystals can be used as standard parameters for further drug development. EF and ROC were used to determine the ideal parameters for the virtual screening process. EF validation and ROC were conducted using $10 \%$ of the compounds that were downloaded from a database of useful (docking) decoysenhanced, which was about 83 actives and 3,505 decoys.

\section{Virtual screening method validation using AutoDock}

Based on the data results, the RMSD of the EGFR target receptor for a $60 \times 60 \times 60$ box using AutoDock does not meet the criteria $(6.076$ from the *.dlg file), so it cannot be used for further virtual screening. The value of cocrystal ligand binding energy was $-6.81 \mathrm{kcal} / \mathrm{mol}$ and the value of the inhibition constant was $10.14 \mu \mathrm{M}$. After validating the cocrystal, virtual screening validation was conducted to control the positive compounds that have been approved by the FDA or have at least entered Phase II of the clinical trials. Table 1 shows the results of positive control virtual screening with AutoDock on the PyRx parameter.

\section{Virtual screening method validation using Vina}

The EGFR cocrystal position with a grid box of 22,500 before and after redocking showed no significant difference based on its RMSD value

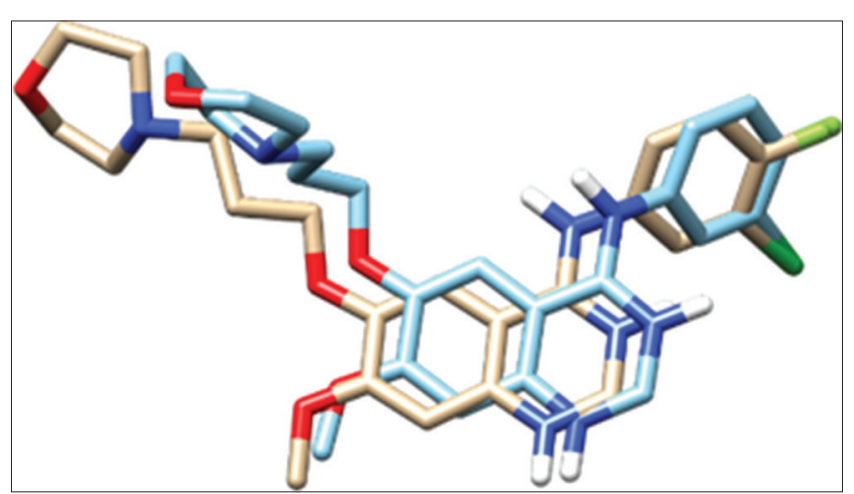

Fig. 1: Superposition result of IRE cocrystal from epidermal growth factor receptor macromolecule using Chimera before (brown) and after (blue) virtual screening on the optimum box of $60 \times 60 \times 60$ using AutoDockTools 
(2.268 using PyMOL), so virtual screening for EGFR macromolecules can be performed using Vina on PyRx. The binding energy was $8.5 \mathrm{kcal} / \mathrm{mol}$. After the validation of the cocrystal, positive control virtual screening optimization was conducted. Table 2 shows the results of the positive control validation using the Vina parameter.

Based on the screening results, EF $1 \%$ (2.61), 10\%, (2.16), and 20\% (1.99) were above the random value ( $>1)$. Ideal EF is $\geq 1$ which shows that the parameter is less prone to an error result (false positive/false negative) [12]. Fig. 2 shows that the ROC curve is above the random line.

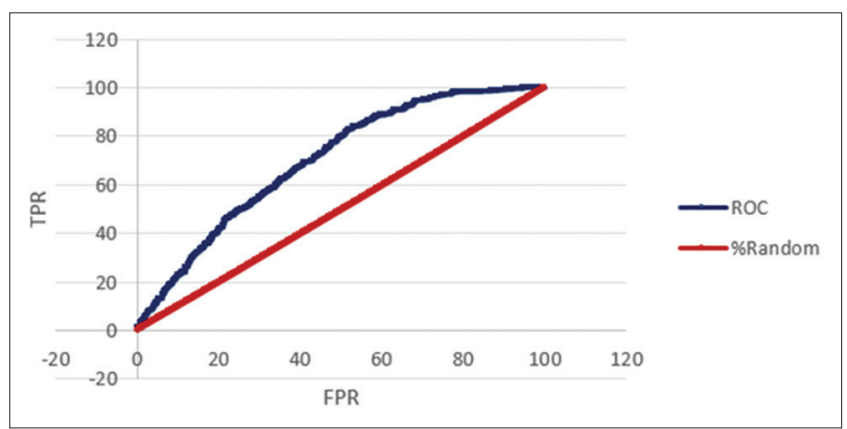

Fig. 2: Receiver operating characteristics curve as the result of epidermal growth factor receptor virtual screening using Vina with a grid box parameter of $22,500 \AA$

Table 1: Virtual screening results of positive control against EGFR target receptor (4WKQ) with AutoDock on the PyRx parameter

\begin{tabular}{ll}
\hline Positive control (FDA) & Binding energy/ $\Delta$ G (kcal/mol) \\
\hline Pazopanib & -9.01 \\
Axitinib & -8.21 \\
Dasatinib & -7.81 \\
Imatinib & -7.64 \\
XL-647 & -7.58 \\
Lapatinib & -7.48 \\
Vandetanib & -7.29 \\
Sorafenib & -7.04 \\
Motesanib & -6.96 \\
Gefitinib & -6.83 \\
BMS-690514 & -6.64 \\
Neratinib & -6.56 \\
Sunitinib & -6.48 \\
Afatinib & -6.15 \\
Erlotinib & -5.82 \\
\hline
\end{tabular}

EGFR: Epidermal growth factor receptor, FDA: Food and Drug Administration

Table 2: Virtual screening results of positive control against EGFR target receptor (4WKQ) with Vina on the PyRx parameter

\begin{tabular}{ll}
\hline Positive control (FDA) & Binding energy/ $\Delta$ G (kcal/mol) \\
\hline XL-647 & -8.8 \\
Imatinib & -8.7 \\
Axitinib & -8.5 \\
Gefitinib & -8.4 \\
Sorafenib & -8.4 \\
Neratinib & -8.3 \\
Vandetanib & -8.3 \\
Motesanib & -8.2 \\
BMS-690514 & -8.1 \\
Sunitinib & -8.1 \\
Afatinib & -8 \\
Pazopanib & -8 \\
Lapatinib & -7.9 \\
Dasatinib & -7.7 \\
Erlotinib & -7
\end{tabular}

The result of the area under a curve calculation using the trapezoidal method is 0.6966. Based on the statement above, the EGFR screening result in a 22,500 $\AA$ grid box using Vina is valid because it meets the criteria ( $>0.5$ ). The ideal ROC value is $>0.5$ (above the random line); the greater the ROC value, the closer it gets to the ideal line [13].

\section{Molecular docking}

We obtained 47 active compounds (ligand) after screening the 268 marine fungi bioactive compounds with EGFR using the Vina parameter. All showed lower to equal binding energy to the EGFR cocrystal; gefitinib with $\Delta \mathrm{G}$ amount of $-8.5 \mathrm{kcal} / \mathrm{mol}$. Further analysis determined the ligand ability to bind to the binding site (AA residues) and their capability of delivering inhibitory activity against these receptors.

\section{Analysis and visualization screening results}

Superposition and bonding analysis of five ranked marine fungi active compounds against EGFR

Table 3: Active compounds as the virtual screening results of marine fungi bioactive compounds to EGFR (4WKQ) target using Vina parameter

\begin{tabular}{|c|c|}
\hline Ligand & Binding energy $/ \Delta G(\mathrm{kcal} / \mathrm{mol})$ \\
\hline FU0202 & -10.4 \\
\hline FU0056 & -10.1 \\
\hline FU0015 & -9.9 \\
\hline FU0051 & -9.9 \\
\hline FU0208 & -9.9 \\
\hline FU0209 & -9.9 \\
\hline FU0017 & -9.8 \\
\hline FU0050 & -9.6 \\
\hline FU0204 & -9.6 \\
\hline FU0203 & -9.4 \\
\hline FU0018 & -9.3 \\
\hline FU0037 & -9.3 \\
\hline FU0091 & -9.3 \\
\hline FU0231 & -9.3 \\
\hline FU0057 & -9.1 \\
\hline FU0107 & -9.1 \\
\hline FU0112 & -9.1 \\
\hline FU0129 & -9.1 \\
\hline FU0198 & -9.1 \\
\hline FU0199 & -9.1 \\
\hline FU0210 & -9.1 \\
\hline FU0048 & -9 \\
\hline FU0115 & -9 \\
\hline FU0229 & -9 \\
\hline FU0038 & -8.9 \\
\hline FU0101 & -8.9 \\
\hline FU0103 & -8.9 \\
\hline FU0193 & -8.7 \\
\hline FU0195 & -8.7 \\
\hline FU0264 & -8.7 \\
\hline FU0014 & -8.6 \\
\hline FU0033 & -8.6 \\
\hline FU0079 & -8.6 \\
\hline FU0126 & -8.6 \\
\hline FU0185 & -8.6 \\
\hline FU0254 & -8.6 \\
\hline FU0036 & -8.5 \\
\hline FU0105 & -8.5 \\
\hline FU0106 & -8.5 \\
\hline FU0113 & -8.5 \\
\hline FU0119 & -8.5 \\
\hline FU0123 & -8.5 \\
\hline FU0125 & -8.5 \\
\hline FU0128 & -8.5 \\
\hline FU0183 & -8.5 \\
\hline FU0184 & -8.5 \\
\hline FU0194 & -8.5 \\
\hline
\end{tabular}




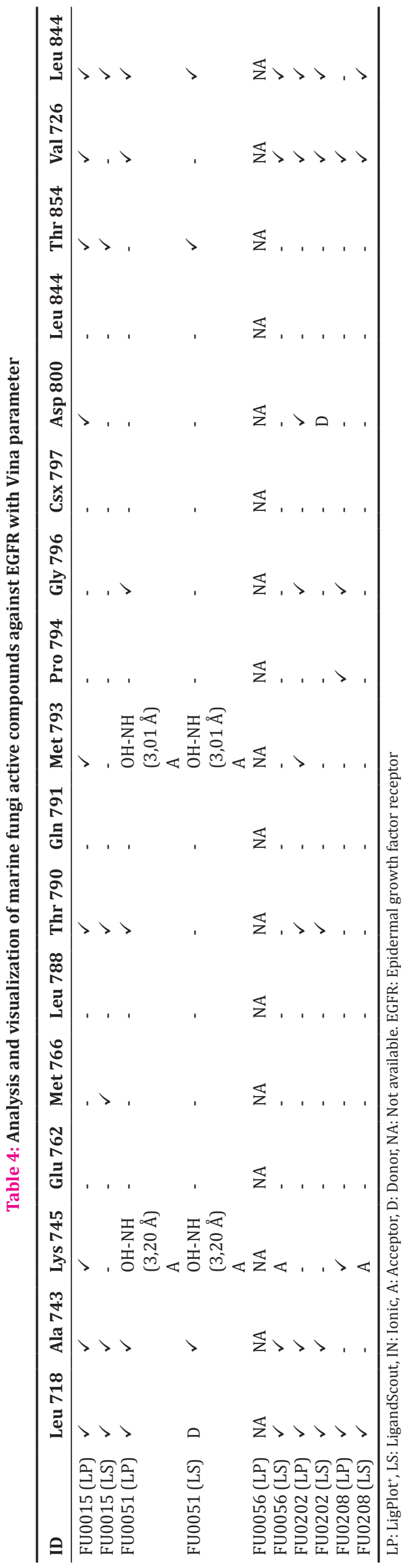

Out of the total number of 47 active compounds resulting from the marine fungi virtual screening using Vina on PyRx, the top five compounds with the lowest binding energy were selected for superposition and bonding analysis. Based on the superposition analysis, FU0051 compounds Fig. 3 show the most similar posing result against the cocrystal, especially on the aromatic ring. While FU0056 and FU0208 show an unfit posing result, FU0056 and FU0208 were

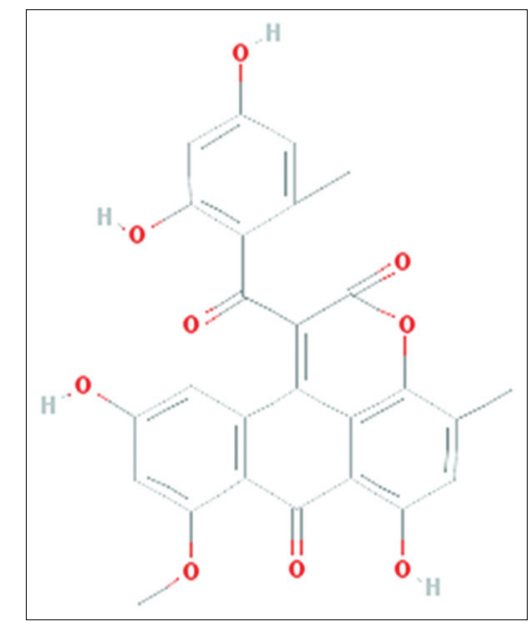

Fig. 3: FU0051

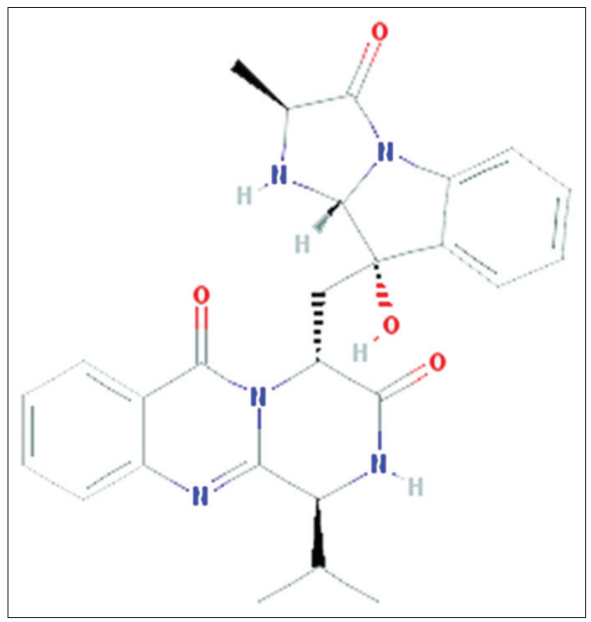

Fig. 4: FU0015

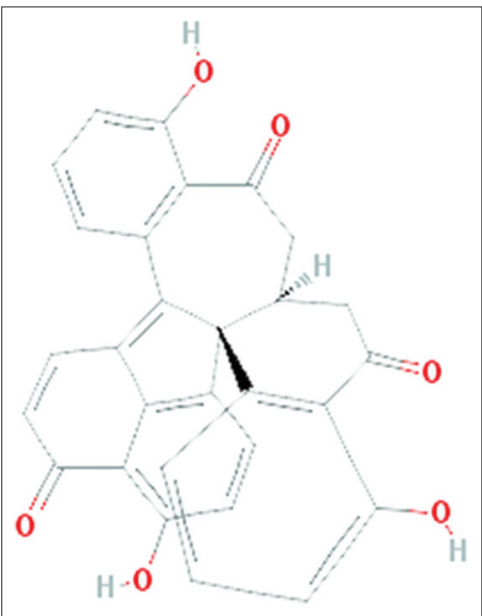

Fig. 5: FU0202 

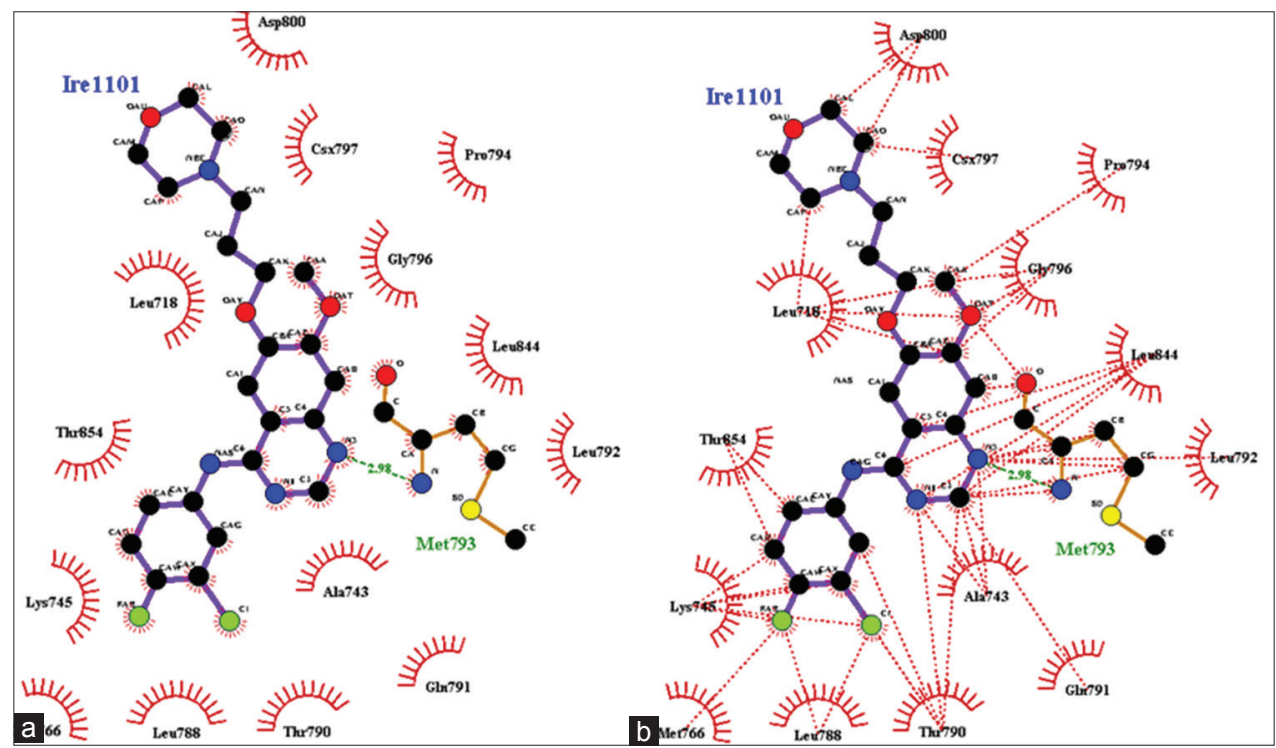

Fig. 6: ( $a$ and b) Interaction analysis and visualization result of cocrystal and positive control against target receptor epidermal growth factor receptor (4WKQ) using LigPlot+ from the Protein Data Bank site

considered as active compounds by regarding binding energy. Further analysis will be presented in the bonding analysis.

Based on the top five compounds, three ligand results were selected as potential inhibitors of EGFR (FU0015, FU0051, and FU0202) (Figs. 3-5). Interaction analysis was conducted to obtain visualization result of cocrystal and positive control against the target receptor EGFR (4WKQ) (Fig. 6)

From the analysis, it can be seen that the majority of the compounds bind to MET-766, THR-790, and THR-854, which proves that these AA residues have potential as active sites for an EGFR-TK inhibitor agent. VAL-726 and LEU-844 AA also seem to always interact with cocrystal ligands, positive control, and the active compounds of marine fungi. Such interactions can be observed when visualized using LigPlot ${ }^{+}$, PyMOL, and LigandScout. Although they are not described as AA residues that interact with ligands on the cocrystal structure sequences by the PDB, both of these residues are hydrophobic, thereby allowing the formation of hydrophobic pockets on the receptor. Their appearance on the AA residues makes them able to have a significant role.

\section{Marine fungi active compounds against EGFR}

So far, this research has concluded that the three marine fungi compounds with the lowest binding free energy, FU0015, FU0051, and FU0202, have great potential as inhibitors of EGFR-TK. Fiscalin A is derived from Neosartorya paulistensis KUFC 7897 (FU0015), Aspergiolide B is derived from Aspergillus flavus (FU0051), and Sporothrix A is derived from Sporothrix sp. (FU0202).

\section{CONCLUSION}

In this research, a database consisting of marine fungi chemical compounds was created by collecting structures from the literature of marine fungi bioactive compounds. The structures were downloaded from PubChem's Open Chemistry Database, ChemSpider Search, and Share Chemistry if they were available or created manually using MarvinSketch if they were unavailable. The total number of molecules obtained was 268. After binding energy analysis, superposition, and bonding analysis, we obtained three potentially active compounds that can be used as inhibitors of EGFR-TK; Fiscalin A, derived from $N$. paulistensis KUFC 7897 (FU0015); Aspergiolide B, derived from A. flavus (FU0051); and Sporothrix A, derived from Sporothrix sp (FU0202).

\section{ACKNOWLEDGMENTS}

The authors would like to acknowledge the financial support of PITTA from Universitas Indonesia and the L'Oréal Sorority in Science.

\section{REFERENCES}

1. da Cunha Santos G, Shepherd FA, Tsao MS. EGFR mutations and lung cancer. Annu Rev Pathol Mech Dis 2011;6(1):49-69.

2. Mosesson Y, Yarden Y. Oncogenic growth factor receptors: Implications for signal transduction therapy. Semin Cancer Biol 2004;14(4):70-262.

3. Ciardiello F, Troiani T, Bianco R, Orditura M, Morgillo F, Martinelli E, et al. Interaction between the epidermal growth factor receptor (EGFR) and the vascular endothelial growth factor (VEGF) pathways: A rational approach for multi-target anticancer therapy. Ann Oncol 2006;17 Suppl 7:vii109-14.

4. Choowongkomon K, Sawatdichaikul O, Songtawee N, Limtrakul J. Receptor-based virtual screening of EGFR kinase inhibitors from the NCI diversity database. Molecules 2010;15(6):4041-54.

5. FDA. FDA Approves Targeted Therapy for First-Line Treatment of Patients with a Type of Metastatic Lung Cancer. FDA News Release; 2015. Available from: http://www.fda.gov/NewsEvents/Newsroom/ PressAnnouncements/ucm454678.htm.

6. Hayashi Y, Yamazaki-Nakamura Y, Yakushiji F. Medicinal chemistry and chemical biology of diketopiperazine-type antimicrotubule and vascular-disrupting agents. Chem Pharm Bull (Tokyo) 2013;61(9):889-901

7. Xiong ZQ, Wang JF, Hao YY, Wang Y. Recent advances in the discovery and development of marine microbial natural products. Mar Drugs 2013;11(3):700-17.

8. Yadav M, Singh G. Bioinformatics: A new era of drug design. Indian J Bioinforma Biotechnol 2013;2(2):48-53.

9. Morris GM, Huey R, Lindstrom W, Sanner MF, Belew RK, Goodsell DS, et al. AutoDock4 and AutoDockTools4: Automated docking with selective receptor flexibility. J Comput Chem 2009;30(16):2785-91.

10. Trott O, Olson AJ. AutoDock Vina: Improving the speed and accuracy of docking with a new scoring function, efficient optimization, and multithreading. J Comput Chem 2010;31(2):455-61.

11. Baber JC, Thompson DC, Cross JB, Humblet C. GARD: A generally applicable replacement for RMSD. J Chem Inf Model 2009:49(8):1889-900.

12. Bender A, Glen RC. A discussion of measures of enrichment in virtual screening: Comparing the information content of descriptors with increasing levels of sophistication. J Chem Inf Model 2005;45(5):1369-75.

13. Triballeau N, Acher F, Brabet I, Pin JP, Bertrand HO. Virtual 
screening workflow development guided by the 'receiver operating characteristic' curve approach. Application to high-throughput docking on metabotropic glutamate receptor subtype 4. J Med Chem 2005;48(7):2534-47.

14. Esteves MS. Anticancer Activity of Marine-Derived Fungi Extracts and Isolated Compounds in Human. Abel Salazar Biomedical Science Institute; 2014. Available from: https://www.repositorio-aberto.up.pt/ bitstream/10216/77834/2/33889.pdf.

15. Gomes NM, Bessa LJ, Buttachon S, Costa PM, Buaruang J, Dethoup T, et al. Antibacterial and antibiofilm activities of tryptoquivalines and meroditerpenes isolated from the marine-derived fungi Neosartorya paulistensis, N. Laciniosa, N. Tsunodae, and the soil fungi N. Fischeri and N. Siamensis. Mar Drugs 2014;12(2):822-39.

16. Dbm V. In silico screening of secondary metabolites derived from marine fungi for anticancer study. J Adv Bioinform Appl Res 2014;5(2):78-82.

17. Zhang JY, Tao LY, Liang YJ, Chen LM, Mi YJ, Zheng LS, et al. Anthracenedione derivatives as anticancer agents isolated from secondary metabolites of the mangrove endophytic fungi. Mar Drugs 2010;8(4):1469-81.

18. Wang X, Mao ZG, Song BB, Chen $\mathrm{CH}$, Xiao WW, Hu B, et al. Advances in the study of the structures and bioactivities of metabolites isolated from mangrove-derived fungi in the South China Sea. Mar Drugs 2013;11(10):3601-16.

19. Bhatnagar I, Kim SK. Immense essence of excellence: Marine microbial bioactive compounds. Mar Drugs 2010;8(10):2673-701.

20. Bhimba BV, Franco DA, Jose GM, Mathew JM, Joel EL. Characterization of cytotoxic compound from mangrove derived fungi Irpex hydnoides VB4. Asian Pac J Trop Biomed 2011;1(3):223-6.

21. Boot CM, Tenney K, Valeriote FA, Crews P. Highly N-methylated linear peptides produced by an atypical sponge-derived Acremonium sp. J Nat Prod 2006;69(1):83-92.

22. Cao F, Yang Q, Shao CL, Kong CJ, Zheng JJ, Liu YF, et al. Bioactive 7-oxabicyclic[6.3.0]lactam and 12-membered macrolides from a gorgonian-derived Cladosporium sp. Fungus. Mar Drugs
2015;13(7):4171-8.

23. Debbab A, Aly AH, Proksch P. Endophytes and associated marine derived fungi-ecological and chemical perspectives. Fungal Divers 2012;57(1):1-39.

24. Debbab A, Aly AH, Lin WH, Proksch P. Bioactive compounds from marine bacteria and fungi. Microb Biotechnol 2010;3(5):544-63.

25. Gomes N, Lefranc F, Kijjoa A, Kiss R. Can some marine-derived fungal metabolites become actual anticancer agents? Mar Drugs 2015;13(6):3950-91

26. Tarman K, Palm GJ, Porzel A, Merzweiler K, Arnold N, Wessjohann LA, et al. A new lactone from an Indonesian marine algicolous strain of Daldinia eschscholzii (Xylariaceae, Ascomycota). Phytochem Lett 2012;5(1):83-6.

27. Li YX, Himaya SW, Dewapriya P, Zhang C, Kim SK. Fumigaclavine C from a marine-derived fungus Aspergillus fumigatus induces apoptosis in MCF-7 breast cancer cells. Mar Drugs 2013;11(12):5063-86.

28. Prompanya C, Fernandes C, Cravo S, Pinto M, Silva A, Kijjoa A, et al. A new cyclic hexapeptide and a new isocoumarin derivative from the marine sponge-associated fungus Aspergillus similanensis KUFA 0013. Mar Drugs 2015;13(3):1432-50.

29. Vasanthabharathi V, Jayalakshmi S. Review on bioactive potential of marine microbes. Afr J Microbiol Res 2013;7(39):4683-8.

30. Tao LY, Zhang JY, Liang YJ, Chen LM, Zhen LS, Wang F, et al. Anticancer effect and structure-activity analysis of marine products isolated from metabolites of mangrove fungi in the South China Sea. Mar Drugs 2010;8(4):1094-105.

31. Tohme R, Darwiche N, Gali-Muhtasib H. A journey under the sea: The quest for marine anti-cancer alkaloids. Molecules 2011;16(11):9665-96.

32. Kim SK. Marine Microbiology: Bioactive Compounds and Biotechnological Applications. Germany: John Wiley \& Sons; 2013.

33. Swathi J. Marine fungal metabolites as a rich source of bioactive compounds. Afr J Biochem Res 2013;7(10):184-96.

34. Yosaatmadja JU, Squire Y, McKeage CJ, Flanagan M. 1.85 angstrom structure of EGFR kinase domain with gefitinib. To Be Publ; 